\title{
Synergistic effect of polymer-surfactant mixtures on the stability of aqueous silica suspensions
}

\author{
Aylin Şakar-Deliormanlı* \\ Izmir Institute of Technology, Chemical Engineering Department, Urla, Izmir, Turkey
}

Available online 14 June 2006

\begin{abstract}
The aim of the present work was to investigate the effect of cationic/nonionic surfactant mixtures on the dispersion and flocculation behavior of aqueous silica suspensions. In the study dodecylamine (DDA) was used as the cationic surfactant and polyethylene oxide-polypropylene oxidepolyethylene oxide (PEO-PPO-PEO) triblock copolymers were employed as the nonionic surfactant. The dispersion and flocculation behavior of aqueous silica suspensions were studied mainly by turbidity measurements at low solids loading $(0.05$ vol.\%) to observe the stability of the system for a given time period. Rheological measurements were performed at higher solids loadings to determine the viscosity as a function of shear rate. Adsorption behavior of single and mixed surfactants onto silica surface was studied using a total organic carbon analyzer.

Results of the study showed that dispersion and flocculation behavior of aqueous silica suspensions depends on the type and concentration of surfactant, cationic/nonionic surfactant ratio and surfactant addition sequence to the system.
\end{abstract}

(C) 2006 Elsevier Ltd. All rights reserved.

Keywords: Suspensions; $\mathrm{SiO}_{2} ;$ Surfactants

\section{Introduction}

Dispersion and flocculation of colloidal suspensions using polymers and surfactants have widespread applications and have a crucial effect in ceramic processing. The production of high-quality ceramics usually requires the preparation of stable suspensions. On the other hand, novel wet shaping methods of ceramics show the importance of investigation on the controlled flocculation of these systems. In other words, in some of the applications destabilization of the oxide particles is the main purpose; however, in other cases stabilization is desired. ${ }^{1}$ Surfactants and polymers can dramatically change the stability of colloidal dispersions because of the interfacial activity and both an increase or decrease of the stability is possible in these systems. When polymers are used their conformation can be manipulated by changing the suspension conditions such as $\mathrm{pH}$ and ionic strength or addition of a second polymer or surfactant may change their activity. Such manipulation can be used to obtain desired levels of flocculation or stabilization. ${ }^{2}$

Several studies have been conducted to understand the adsorption mechanism from single or mixed surfactant solu-

\footnotetext{
* Tel.: +90 232 7506680; fax: +90 2327506196.

E-mail address: aylindeliormanli@iyte.edu.tr.
}

tions in relation to stability of metal oxide/water systems. ${ }^{3,4}$ But there is a special interest for the investigation of ionic/nonionic surfactant mixtures. Effects related to competitive or synergistic adsorption of anionic and nonionic surfactants were observed in the flocculation studies of alumina by Esumi et al. ${ }^{5}$ It was shown that the co-adsorption behavior at the solid liquid interface depends on the molecular structures of the co-adsorbates. For the dual systems, when the interaction between the surfactants is favorable, enhancement of adsorption of one surfactant would occur by the adsorption of another surfactant. Similarly Fan et al. have studied the co-adsorption of anionic and nonionic surfactants on and alumina. ${ }^{6}$ It was found that the sequence of addition is crucial in determining the dispersion and flocculation behavior. Palla and Shah have been shown that the synergistic mixtures of ionic and nonionic surfactants are capable of stabilizing suspensions in which either ionic or nonionic surfactants are insufficient stabilizers. ${ }^{7}$ As discussed by the same scientists, this kind of stabilization process is dependent on the choice of ionic and nonionic surfactant. As a consequence in literature the use of mixed surfactant solutions often results in enhanced performance and it is a synergistic process. ${ }^{7,8}$

Water soluble polyethylene oxide-polypropylene oxidepolyethylene oxide PEO-PPO-PEO block copolymers are characterized by good stabilization ability and have widespread 


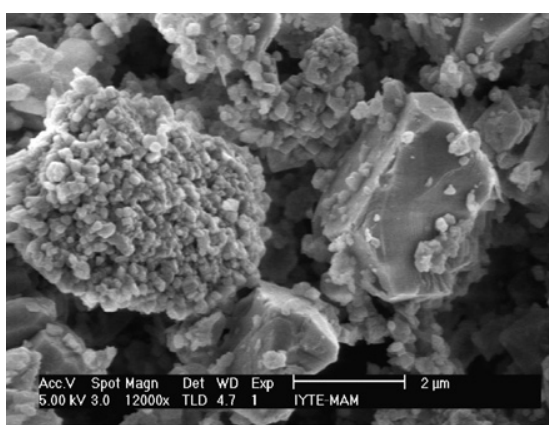

(a)

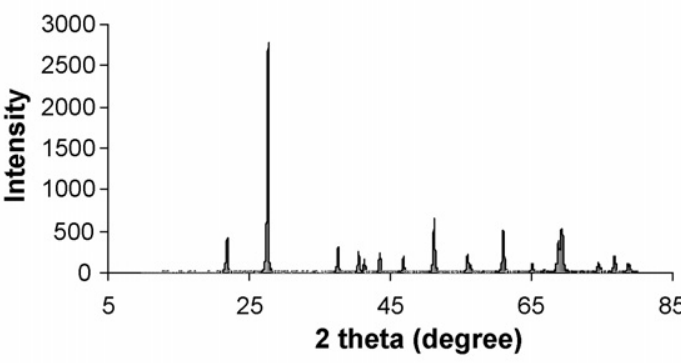

(b)

Fig. 1. (a) SEM micrograph and (b) X-ray diffraction pattern of the silica powder.

Table 1

Important properties of the block copolymers used in the study

\begin{tabular}{llllll}
\hline Code & Mw & Composition & PEO $(\%)$ & Melt point $\left({ }^{\circ} \mathrm{C}\right)$ & Viscosity (cps) \\
\hline F127 & 12,600 & $\mathrm{EO}_{97} \mathrm{PO}_{69} \mathrm{EO}_{97}$ & 70 & 56 & 3100 \\
F68 & 8400 & $\mathrm{EO}_{78} \mathrm{PO}_{30} \mathrm{EO}_{78}$ & 80 & 52 & 1000 \\
\hline
\end{tabular}

applications. They consist of two hydrophilic PEO chains and a hydrophobic PPO chain and is available in different segment lengths. Although the adsorption behavior of PEO homopolymers and PEO-PPO-PEO triblock copolymers onto silica surface and rheological behavior of these systems have been investigated previously, combined effect of these polymeric surfactants with the simple cationic surfactants have not been studied in detail. $^{9-13}$ Therefore in this study it was intended to investigate the effects of PEO-PPO-PEO type block copolymer and dodecylamine DDA alone and as a mixture on the stability of aqueous silica suspensions.

\section{Experimental}

\subsection{Materials}

A commercially available silica powder, from Sigma-Aldrich Chemicals (S5631) was used in the study. The powder purity is approximately $99 \%$ and it has a wide particle size distribution. $80 \%$ of the powder is between $1-5 \mu \mathrm{m}$ as reported by the manufacturer company. The specific surface area of the powder was measured to be $6.29 \mathrm{~g} / \mathrm{m}^{2}$ using ASAP 2400 Micromeritics Instrument, USA by nitrogen adsorption with multi point BET method on samples degassed at $150^{\circ} \mathrm{C}$. Isoelectric point (iep) of the silica powder was found to be at $\mathrm{pH} 2.9$ using Zetasizer 3000 HSA, Malvern Instruments, UK. Fig. 1 shows the microstructure and the X-ray diffraction pattern of the silica powder obtained by scanning electron microscope, Phillips XL-30S FEG and X-ray diffraction machine Phillips X'Pert Pro, respectively.

Triblock copolymers (Pluronic F127 and F68) used in the study were obtained from BASF Corporation, Washington, NJ, USA. Some properties of these polymers are tabulated in Table 1. The cationic surfactant, dodecylamine was obtained from Aldrich Chemicals with Mw $185.4 \mathrm{~g} / \mathrm{mol}$ and purity $98 \%$. Chemical structure of the surfactants used in the study is shown in Fig. 2. Deionized water with a resistivity of $18.2 \mathrm{M} \Omega \mathrm{cm}$ was used throughout the experiments. Adjustment of the $\mathrm{pH}$ was performed using analytical grade $\mathrm{HCl}$, Merck Chemicals.

\subsection{Method}

In the study suspensions were prepared at different solids loadings namely $0.05,10$, and 30 vol.\%. Suspension preparation and characterization methods summarized in the following paragraphs.

\subsubsection{Turbidity measurements}

Suspensions were prepared at 0.05 vol. $\%$ using two methods. In the first method silica powder was added into surfactant solution and stirred for 15 min using magnetic stirrer then 10 min of ultrasonic treatment and $15 \mathrm{~min}$ of stirring were applied subsequently. In the second method, suspensions were stirred for $24 \mathrm{~h}$.
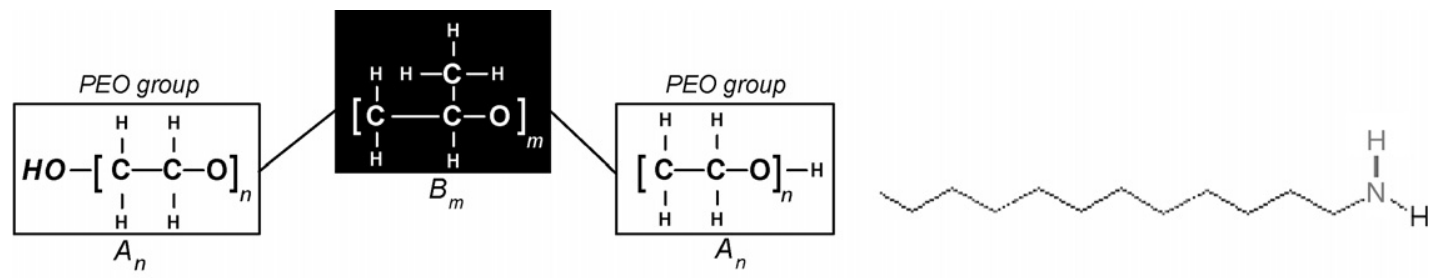

(a)

(b)

Fig. 2. Chemical structure of (a) PEO-PPO-PEO tri block copolymers (Pluronic F-127, F-68); and (b) dodecylamine. 
Measurements were conducted in the range $30 \mathrm{~s}-64$ min using a Turbidimeter WTW 555, Germany. Turbidity is an optical property that causes light to be scattered and absorbed rather than transmitted in straight lines through the sample. A turbidimeter measure the light scattered at an angle to the incident light beam and then relate this angle scattered light to the samples turbidity. Most modern instruments measure $90^{\circ}$ scatter and they are called nephelometric turbidimeter. Turbidity measured in this way is stated in nephelometric turbidity units (NTU). ${ }^{14}$ In the current study, turbidity measurements were performed using nephelometric ratio method and results were recorded in terms of NTU with \pm 2 of value or \pm 0.01 NTU accuracy.

\subsubsection{Rheological measurements}

Rheological properties of the suspensions (30 vol.\%) were analyzed using a Brookfield DV-III Programmable rheometer (Brookfield, USA) with a UL adaptor and a concentric cylinder measuring unit. Suspensions were mixed for 1 week using a laboratory shaker (Gerhardt-Germany, THO 500, Incubator shaker) at a shaking frequency of $150 \mathrm{~min}^{-1}$ and temperature $25^{\circ} \mathrm{C}$ before the measurements. Viscosities and shear stresses of the suspensions were measured at different shear rates under the following conditions:

- Initial shear rate was set at $10 \mathrm{~s}^{-1}$.

- It was increased to $250 \mathrm{~s}^{-1}$ by $10 \mathrm{~s}^{-1}$ in every $30 \mathrm{~s}$.

- All measurements were performed at $25( \pm 1)^{\circ} \mathrm{C}$.

\subsubsection{Adsorption studies}

For the adsorption studies the suspensions (10 vol.\%) were homogenized and stirred for 1 week to ensure the equilibrium using a laboratory shaker (Gerhardt-Germany, THO 500, Incubator shaker) at a shaking frequency of $150 \mathrm{~min}^{-1}$ and temperature $25^{\circ} \mathrm{C}$. Each suspension was then centrifuged at $3000 \mathrm{rpm}$ for $1 \mathrm{~h}$ and the supernatant solution was analyzed using a total organic carbon analyzer (Shimadzu TOC-V CPH, Japan). TOC analyses were performed in triplicate. The amounts of surfactant adsorbed obtained from the difference between the initial surfactant concentration and the equilibrium concentration measured in the supernatant. The adsorption behavior on silica was determined by comparing the data against known calibration curves.

\section{Results and discussion}

\subsection{Stability of the system by turbidity measurements}

Turbidity measurements were conducted to study the stability of silica/water system at low solids loading in the absence and presence of surfactants F127, F-68 and DDA. Fig. 3 shows the effect of $\mathrm{pH}$ on the stability of the silica/water and sil$\mathrm{ica} /$ water/F127 suspensions. The results showed that the $\mathrm{pH}$ of the medium is very effective concerning flocculation behavior. As expected particles settled around $\mathrm{pH} 2$, which is very close to the isoelectric point of silica. Natural $\mathrm{pH}$ values of the suspensions were in the range of $6.5-7.5$ and at these $\mathrm{pH}$ values turbidity values were high.

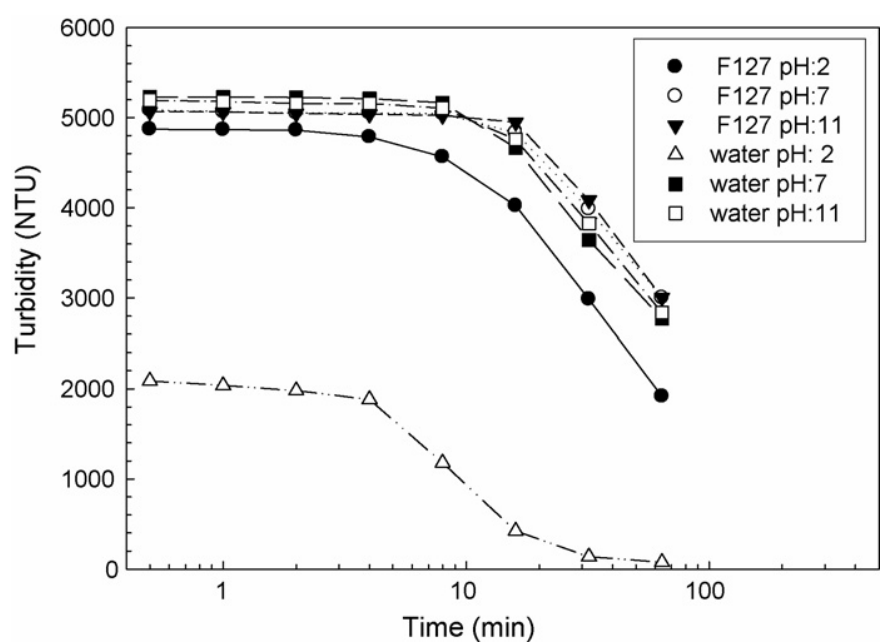

Fig. 3. Effect of $\mathrm{pH}$ on the stability of aqueous silica suspensions in the absence and presence of nonionic block copolymer F127 at $10^{-3} \mathrm{M}$.

Fig. 4 shows the effect of F127 and DDA alone on the stability of the aqueous silica suspensions for short mixing times (30 min). According to the results F127 provides a good dispersion; however, DDA cause flocculation at all concentrations. Similarly Wang et al. showed that the addition of DDA at $3.75 \times 10^{-4} \mathrm{Mm}$ into a fine silica suspension at $\mathrm{pH} 8$ caused significant aggregation and rapid settling of silica particles. ${ }^{15}$ This is most probably due to the adsorption of positively charged DDA onto negatively charged silica particles. Adsorption is expected to occur by electrostatic attraction and orientation of the hydrocarbon chain of the DDA molecules is towards the aqueous solution. Therefore, silica surface becomes hydrophobic and particles attract each other to flocculate.

On the other hand, turbidity values of the suspensions prepared by longer mixing time $(24 \mathrm{~h})$ was not the same with the turbidity values presented in Fig. 4. In this case again DDA at $10^{-3}$ and $10^{-4} \mathrm{M}$ caused a strong flocculation the system. But also a decrease was observed in the turbidity values of the silica suspensions in the presence of F127 (Fig. 10).

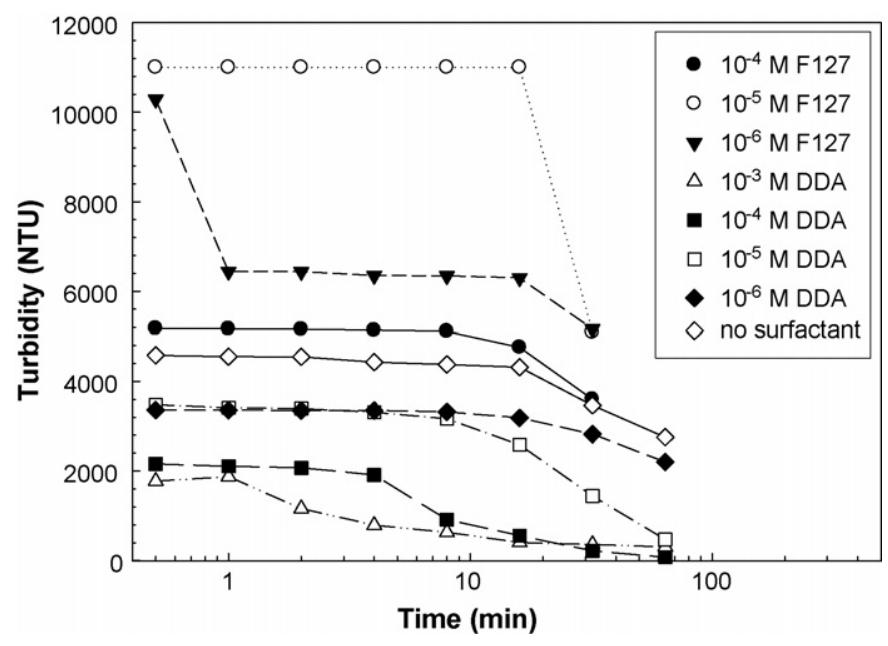

Fig. 4. Turbidity values of silica suspensions in presence of single surfactants, F127 and DDA, pH 7. 


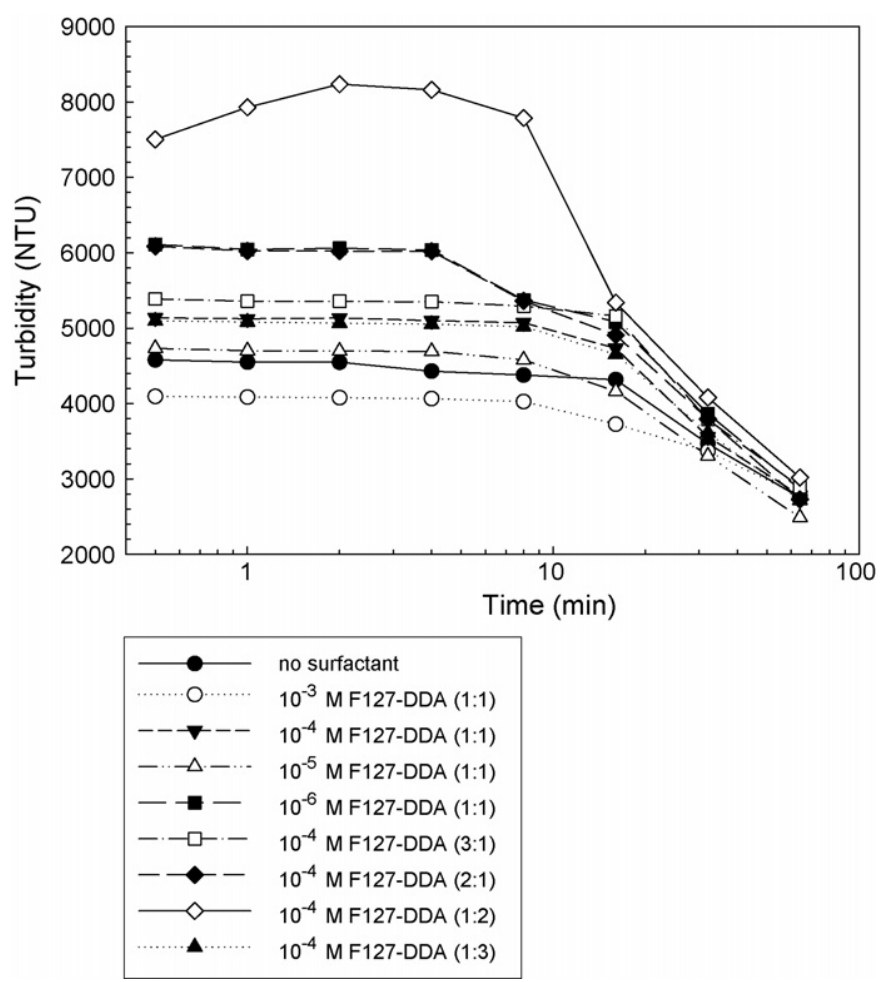

Fig. 5. Turbidity values of the silica suspensions in the presence of DDA-F127 mixture. Simultaneous addition with nonionic-ionic surfactant ratio $(x: y), \mathrm{pH} 7$.

\subsubsection{Simultaneous addition}

Fig. 5 shows the effect of simultaneous addition of F127 and DDA on the stability of the system. Results indicate that simultaneous addition of F127 and DDA in an equal ratio (1:1) has a positive effect on the stability of the system for short stirring times. Only at high surfactant concentration $\left(10^{-3} \mathrm{M}\right)$ flocculation was observed. But as the stirring time increased since the adsorption equilibrium was reached the simultaneous addition of F127 and DDA caused flocculation almost at all concentrations.

The effect of the surfactant ratio on the stability of silica suspensions was also tested in the study. Although very high turbidity values were obtained in the case of F127 and DDA mixture at (1:2) ratio, after 16 min nearly all the surfactant mixtures having different ionic/nonionic ratios followed a similar pattern.

In the study effect of lower molecular weight nonionic F68 and cationic DAA on the stability of silica suspensions were also investigated (Fig. 6). The stability of the aqueous silica suspensions containing F68 showed similar behavior within care of F127 addition but having higher turbidity values. Turbidity values in the presence of $10^{-4} \mathrm{M}$ F-68 were 11000 NTU up to $64 \mathrm{~min}$. Since the F68 structure has a higher content in PEO than F127, the higher stability provided by the F68 may be attributed to this feature. Additionally, the mixture of F68 and DAA with equal ratios at $10^{-5} \mathrm{M}$ again provided good stability.

\subsubsection{Preferential Addition}

In the study the effects of the addition mode of the mixed surfactants on the stability of the silica suspensions was studied using turbidity measurements. Fig. 7 illustrates the effect of

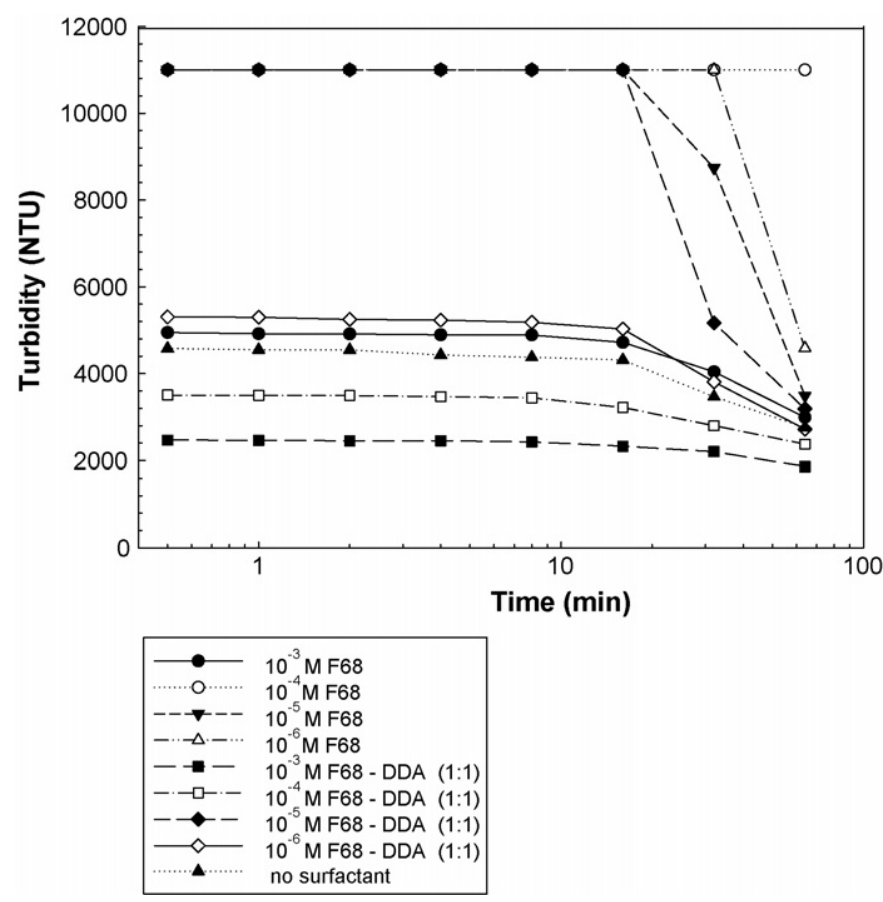

Fig. 6. Turbidity values of aqueous silica suspensions in the presence of F68 and DDA-F68 mixtures; Simultaneous addition with nonionic-ionic surfactant ratio $(x: y), \mathrm{pH} 7$.

sequential addition of surfactants on the stability of the system for short mixing times ( $30 \mathrm{~min}$ ). According to Fig. 7 when F127 is added to the system before DDA it provides higher stability at $10^{-4}$ and $10^{-5} \mathrm{M}$ but at high polymer concentrations, such

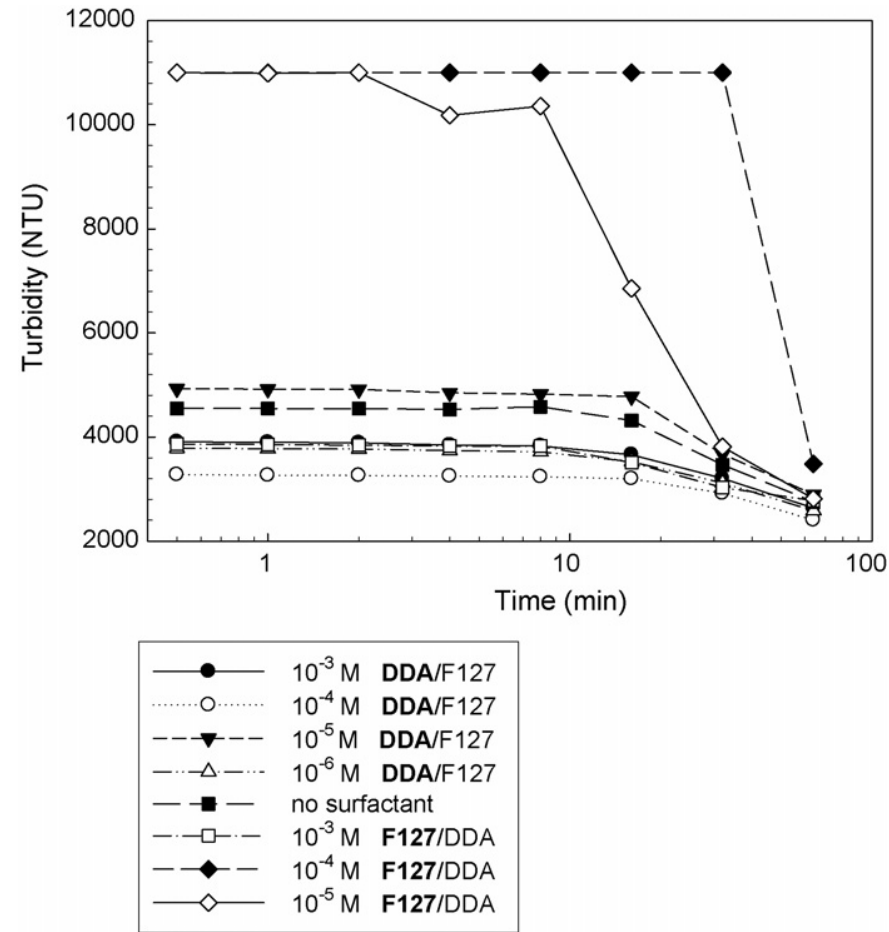

Fig. 7. Turbidity values of aqueous silica suspensions in the presence of surfactant mixtures; sequential addition with nonionic/ionic surfactant ratio (1:1), 30 min mixing, $\mathrm{pH} 7$. 


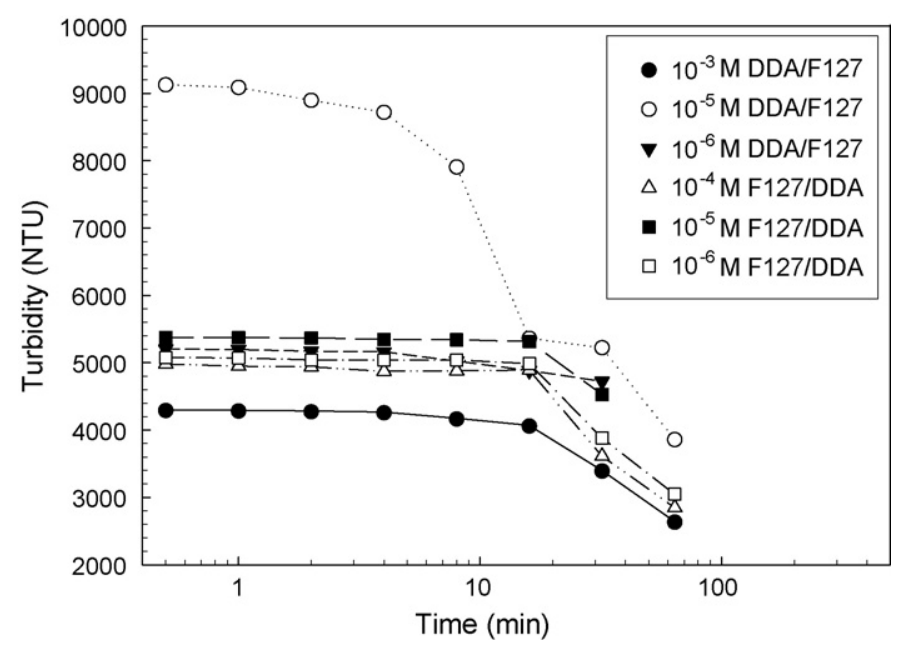

Fig. 8. Turbidity values of silica suspensions in the presence of surfactant mixtures; sequential addition with nonionic/ionic surfactant ratio (1:1), $24 \mathrm{~h}$ mixing, pH 7.

as $10^{-3} \mathrm{M}$ it causes flocculation since this value is close to the critical micelle concentration of F127.

However, the behavior of the same system after longer mixing periods (24 hours) is not the same (Fig. 8). In this case higher stability was achieved when DDA was added to the system first followed by F127 addition. Especially at $10^{-5} \mathrm{M}$ initial addition of DDA provided very high turbidity values in the silica/water system. The mechanism of the stability provided by the sequential addition of surfactants can be explained by the hydrocarbon chain interaction between cationic DDA and nonionic F127. When DDA is added to the system first it attaches to the silica surface by hydrophilic amine groups and creates highly hydrophobic surface sites on silica due to the hydrophobic dodecyl groups. On the other hand, tri-block copolymers have hydrophilic PEO groups and hydrophobic PPO groups in their structure (see Fig. 2). A possible stabilization mechanism may be the hydrocarbon chain interaction of hydrophobic PPO groups of the copolymers with hydrophobic groups of the DDA molecules. By this way hydrophilic PEO groups stay in the solution and provide larger steric barrier between the silica particles. This mechanism is shown schematically in Fig. 9.

\subsubsection{Effect of stirring time}

Results showed that the stability of the silica/water system in presence of surfactants is dependent on the stirring time of the suspensions. According to the results presented in Fig. 10 in the case of short stirring times $(30 \mathrm{~min})$ depletion stabilization may be dominant. It is well-known that sterically stabilized suspensions in the presence of unadsorbed polymers display depletion effects, which can induce either flocculation or stabilization. Depletion interactions are governed by the balance between the osmotic pressure exerted on the particles by the polymer solution and the size of the depletion region from which the macromolecules are expelled. ${ }^{9}$

In the case of long stirring times block copolymers adsorb onto the silica leading to a hydrophobic surface. This is indicated (a)

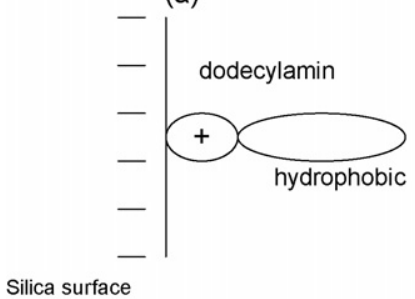

(c)

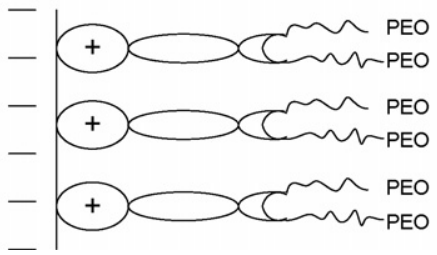

Fig. 9. Schematic illustration of the stabilization mechanism provided by the surfactant mixtures.

by the low turbidity values. It is also well-established that the adsorption of polymers on most surfaces is irreversible resulting in the so called high affinity adsorption. ${ }^{16}$ Therefore, the decrease of the turbidity values after longer stirring time cannot be explained by the desorption mechanism. Fig. 10 shows the effect of mixing time on the stability of silica suspensions in the presence of F127.

Results of the current study also agree well with the findings of a previous study which was carried by Whitby et al. ${ }^{17}$ According to the results of this previous study adsorbed PEO layers impart steric stabilization to silica dispersions. Some evidence was also found for depletion interactions in the presence of significant concentrations of unadsorbed PEO. At low PEO coverage of silica, attractive bridging forces were measured between silica surfaces while at higher coverage, repulsive steric interactions were observed. ${ }^{17}$

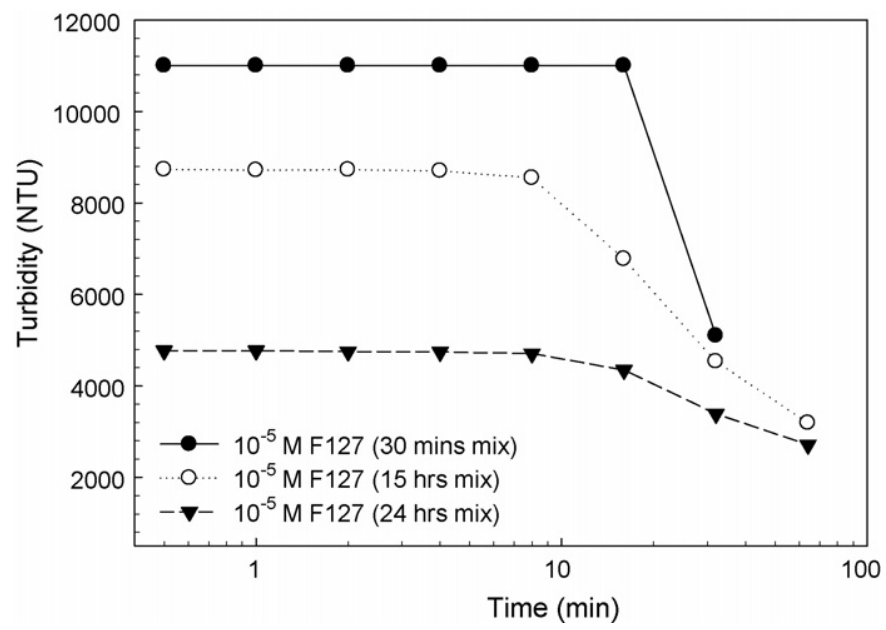

Fig. 10. Effect of stirring time on the stability of aqueous silica suspensions in the presence of $\mathrm{F} 127$ at $\mathrm{pH} 7$. 


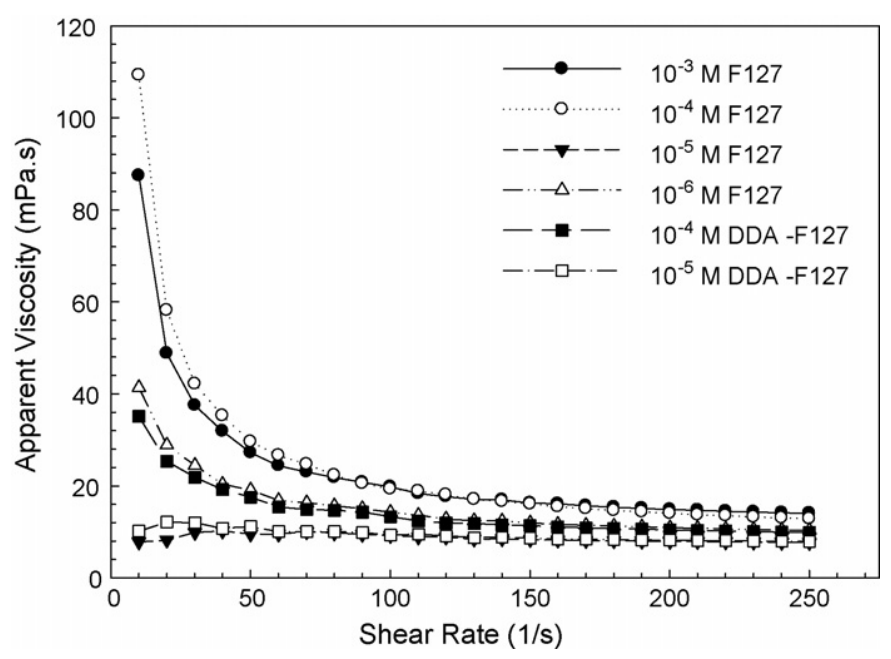

Fig. 11. Viscosity vs. shear rate graph for aqueous silica suspensions in the presence of F127 and DDA-F127 mixture. Simultaneous addition with nonionicionic surfactant ratio (1:1), $\mathrm{pH} 7$, solids loading $30 \mathrm{vol} . \%$.

\subsection{Rheological measurements}

The stability of the silica suspensions could be related to the rheological properties of this suspensions. In the current study rheological behavior of the aqueous silica suspensions in the absence and presence of surfactants were studied at 30 vol.\% solids loading and $\mathrm{pH} 7$. Typical examples of the flow curves are given in Figs. 11 and 12. It is interesting to observe that simultaneous addition of DDA-F127 mixture at (1:1) ratio to the silica/water system provided lower viscosities at $10^{-4}$ and $10^{-5} \mathrm{M}$ compared to the values of the suspensions in the presence of single surfactants. The flow characteristics of the suspensions containing $10^{-3}$ and $10^{-4} \mathrm{M}$, nonionic F127 fits with the Bingham flow model with a yield stress 0.93 and $0.55 \mathrm{~N} / \mathrm{m}^{2}$, respectively. The similar behavior was observed also for the suspensions containing DDA-F68 mixture at $(1: 1)$ ratio. At certain concentrations $\left(10^{-5}, 10^{-4} \mathrm{M}\right)$ simultaneous addition of F68

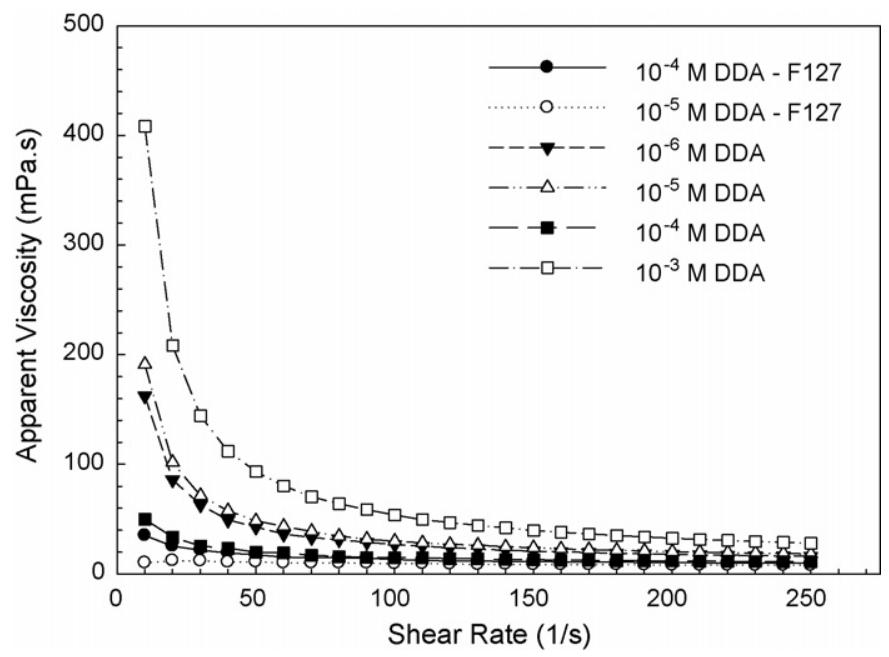

Fig. 12. Viscosity vs. shear rate graph for aqueous silica suspensions in the presence of DDA and DDA-F127 mixture. Simultaneous addition with nonionicionic surfactant ratio (1:1), pH 7, solids loading $30 \mathrm{vol} \%$. and DAA mixture to the silica/water system provided very low viscosity values.

On the other hand, according to Fig. 12, the flow curve of the aqueous silica suspension containing DDA at $10^{-3} \mathrm{M}$ fits with the Casson Model (yield stress $2.77 \mathrm{~N} / \mathrm{m}^{2}$ ). At lower DDA concentrations such as $10^{-5}$ and $10^{-6} \mathrm{M}$ Bingham flow model was consistent. Yield stresses observed in the Figs. 11 and 12 may be attributed to the wider particle size distribution or the rough non-spherical morphology of the quartz particles. Flow characteristics of the suspensions can be explained by the formation of flocs in the structure. When the attractive forces between particles prevail, collisions among them will lead to the formation of agglomerates. At low shear rates, liquid is immobilized in void spaces within flocs and floc network. As the shear rate is increased, the flocs break down the entrapped liquid is released and a more ordered structure in the flow direction is formed. ${ }^{18}$

\subsection{Adsorption studies}

The results of the adsorption studies of single and mixed surfactants DDA, F127 and F68 onto silica surface is presented in Fig. 13. Results showed that adsorption of PEO-PPO-PEO onto silica surface depends on the molecular weight and the PEO/PPO ratio (Fig. 13). Higher molecular weight of a copolymer means higher flexibility of the entire molecule. This increase flexibility offers a better protection of the PPO block (toward to the aqueous environment). But if we compare the adsorption behavior of F127 and F68 as it is seen in Fig. 13, F68 with lower molecular weight and higher PEO portion (PEO/PPO: 80/20) showed higher adsorption onto silica surface than the F127.

Hydrogen bonding between the ether oxygen and the silanol groups of the silica surface is responsible for the adsorption of the block copolymers onto the silica surface. In a previous study Kawaguchi et al. ${ }^{10}$ explained the adsorption of the same type of block copolymers by the hydrogen bonding between the ether oxygen and the silanol groups of the silica surface leading to suppression of an aggregated structure of the silica particles, in water maintained through interaction between silanol groups on the silica surface. Calorimetric data for the adsorption of nonionic surfactant nonyl phenol ethoxylate on silica suggest adsorption with part of their oxyethylene head on the solid and the other part in the bulk. Spectroscopic evidence exits for hydrogen bonding between polyethylene oxide groups from nonionic surfactants and the hydroxyl groups at the silica surface. ${ }^{19}$

In the current study, adsorption of positively charged DDA onto negatively charged silica was studied in the same way with the block copolymers. Especially at high concentrations such as $10^{-3} \mathrm{M}$, DDA created highly hydrophobic surfaces. Wang et al., showed the enhance transfer of DDA molecules onto the silica surface by surface tension measurements. ${ }^{8}$ A similar surface tension was observed with and without centrifuging the silica suspensions, confirming a negligible transfer of the adsorbed surfactant from the solid surface. However, in the current study results showed that adsorption of $10^{-3} \mathrm{M}$ DDA onto silica surface was not negligible.

According to Fig. 13, highest adsorption percentage was obtained in the case of simultaneous addition of DAA-F127 or 

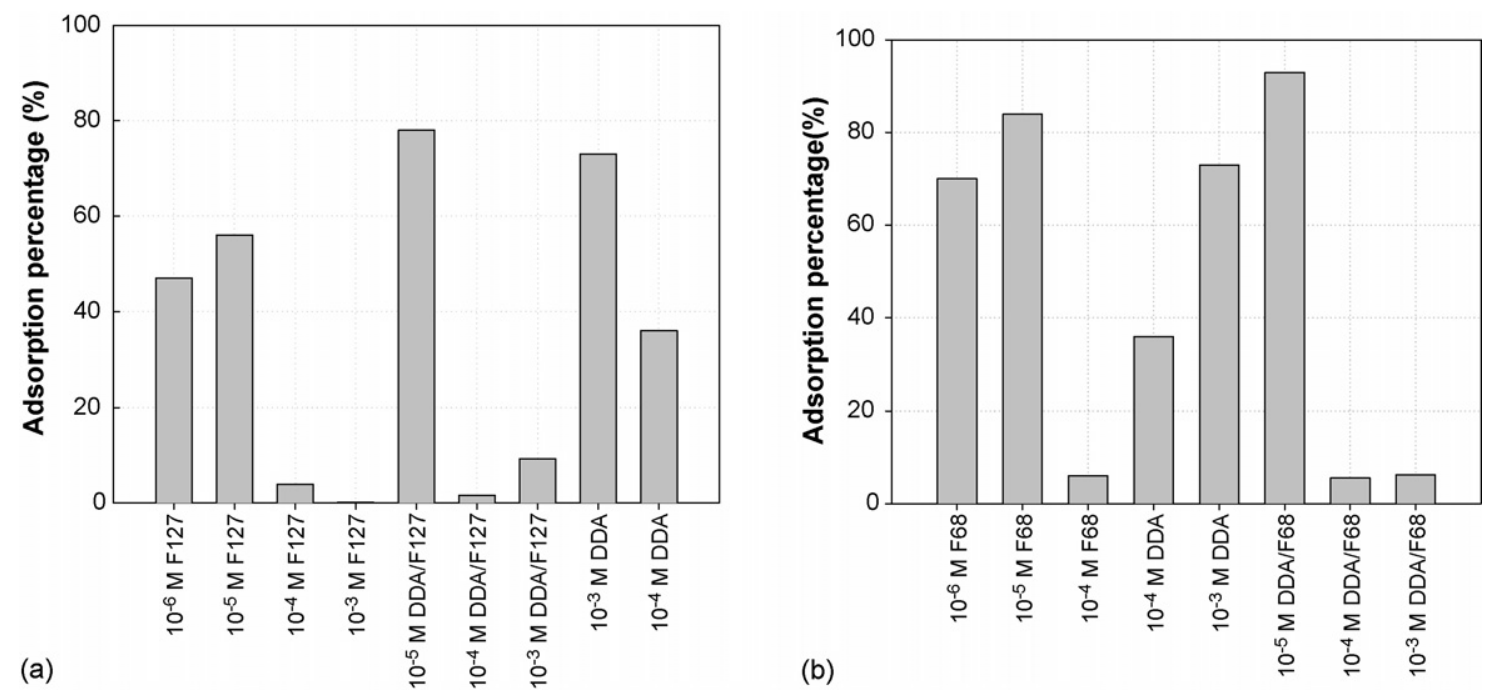

Fig. 13. Adsorption percentage of ionic and nonionic surfactants and surfactant mixtures (a) DDA-F127 (b) DDA-F68 onto silica surface, pH 7, solids loading 10 vol. $\%$, temperature $25^{\circ} \mathrm{C}$.

DAA-F68 mixture at $10^{-5} \mathrm{M}$ and (1:1) ratio to the silica/water system. In the same way stability of these systems was high as discussed in the previous sections. The possible stabilization mechanism provided by the ionic/nonionic surfactant mixtures used in this study is shown in Fig. 9. According to this mechanism first DDA adsorb onto silica surface with full surface coverage via the electrostatic attractions and make silica surface hydrophobic due to dodecyl group. It is followed by the nonionic surfactant adsorption due to PPO group onto DDA molecules via hydrophobic effect. The stabilization is brought by the steric barrier created by the hydrophilic PEO group of the nonionic surfactant extending into the solution. ${ }^{7,20}$

\section{Conclusions}

- The effect of dodecylamine/block copolymer mixtures on the dispersion/flocculation behavior of aqueous silica suspensions was studied. Turbidity values of the silica suspensions in the presence of single block copolymers showed a time dependent behavior. Very high turbidity values of silica suspensions containing block copolymers in the first time interval was attributed to the depletion stabilization mechanism. As the time proceeds silica surface became hydrophobic and the turbidity values lowered. Similar effect was observed in the case of suspensions containing cationic surfactant/block copolymer mixture at different ratios.

- The cationic surfactant/block copolymer addition sequence strongly affected the stability of the system. Higher turbidity values were obtained when DDA was added to the system first followed by F127 or F68 addition.

- Adsorption studies showed that adsorption of surfactants onto silica surface has a maximum at $10^{-5} \mathrm{M}$ for F-127 and F68 and at $10^{-3} \mathrm{M}$ for DDA, respectively. The adsorption amount increases in the case of suspensions containing DAA-F127 and DAA-F68 mixtures.
- A possible stabilization mechanism of ionic/nonionic mixtures in silica/water system is the adsorption of block copolymers to cationic surfactant due to strong hydrophobic interactions, which also enhance the stability of the system and have synergistic effects.

\section{Acknowledgements}

Partial financial support by The Scientific and Technological Research Council of Turkey is acknowledged. The author would like to thank the Environmental Research Center at Izmir Institute of Technology for use of the total organic carbon analyzer. Invaluable advice and discussions with Dr. Hurriyet Polat is gratefully acknowledged.

\section{References}

1. Bijsterbosch, H. D., Stuart, C. M. A. and Fleer, G. J., Effect of block and graft copolymers on the stability of colloidal silica. J. Colloid Interface Sci., 1999, 210, 37-42.

2. Somasundaran, P., Yu, X. and Krishnakumar, S., Role of conformation and orientation of surfactants and polymers in controlling flocculation and dispersion of aqueous and non-aqueous suspensions. Colloids Surf. A: Physicochem. Eng. Aspects, 1998, 133, 125-133.

3. Bremmell, K. E., Jameson, G. J. and Biggs, S., Polyelectrolyte adsorption at the solid/liquid interface interaction forces and stability. Colloids Surf. A: Physicochem. Eng. Aspects, 1998, 139, 199-211.

4. Xu, Q. and Vasudevan, T. V., Stabilization of kaolinite suspensions by anionic-nonionic surfactant mixtures. J. Disper. Sci. Technol., 1991, 12, 83-93.

5. Esumi, K., Iitaka, M. and Torigoe, K., Kinetics of simultaneous adsorption of poly(vinylpyrrolidone) and sodium dodecyl sulfate on alumina particles. J. Colloid Interface Sci., 2000, 232, 71-75.

6. Fan, A., Somasundaran, P. and Turro, N. J., Role of sequential adsorption of polymer/surfactant mixtures and their conformation in dispersion/flocculation of alumina. Colloids Surf. A: Physicochem. Eng. Aspects, 1999, 146, 397-403.

7. Palla, B. J. and Shah, D. O., Stabilization of high ionic strength slurries using surfactant mixtures: molecular factors that determine optimal stability. $J$. Colloid Interface Sci., 2002, 256, 143-152. 
8. Wang, W. and Kwak, J. C. T., Adsorption at the alumina-water interface from mixed surfactant solutions. Colloids Surf. A: Physicochem. Eng. Aspects, 1999, 156, 95-110.

9. Neel, O., Ducouret, G. and Lafuma, F., Fludification of concentrated aqueous colloidal silica suspensions by adsorption of low molecular weight poly(ethylene oxide). J. Colloid Interface Sci., 2000, 230, 224-253.

10. Kawaguchi, M., Yamamoto, T. and Kato, T., Rheological properties of silica suspensions in aqueous solutions of block copolymers and their watersoluble components. J. Colloid Interface Sci., 2001, 241, 293-295.

11. Şakar-Deliormanlı, A., Polat, H. and Çiftçioğlu, M., Alumina/water suspensions in the presence of PEO/PPO/PEO triblock copolymers. J. Eur. Ceram. Soc., 2004, 24, 3063-3072.

12. Zaman, A. A., Bjelopavlic, M. and Moudgil, B. M., Effect of adsorbed polyethylene oxide on the rheology of colloidal silica suspensions. J. Colloid Interface Sci., 2000, 226, 290-298.

13. Gosa Lisette, K. and Uricanu, V., Emulsions stabilized with PEO-PPO-PEO block copolymers and silica. Colloids Surf. A: Physicochem. Eng. Aspects, 2002, 197, 257-269.

14. Sadar, M. J., Turbidity Science, Technical Information Series Booklet, No 11. Hach Company, USA, 1998.
15. Wang, W., Zhou, Z., Nandakumar, K., Xu, Z. and Masliyah, J. H., Effect of charged colloidal particles on adsorption of surfactants at oil-water interface. J. Colloid Interface Sci., 2004, 274, 625-630.

16. Rachas, I., Tadros, T. F. and Taylor, P., The displacement of adsorbed polymer from silica surfaces by the addition of a nonionic surfactant. Colloids Surf. A: Physicochem. Eng. Aspects, 2000, 161, 307319.

17. Whitby, C. P., Scales, P. J., Grieser, F., Healy, T. W., Kirby, G., Lewis, J. A. and Zukoski, C., PAA/PEO comb polymer effects on the rheological properties and the interparticle forces in aqueous silica suspensions. J. Colloid Interface Sci., 2003, 262, 274-281.

18. Olhero, S. M. and Ferreira, J. M. F., Influence of particle size distribution on rheology and particle packing of silica based suspensions. Powder Technol., 2004, 139, 69-75.

19. Colic, M., Fisher, M. L. and Fuerstenau, D. W., Electrophoretic behavior and viscosities of metal oxides in mixed surfactant systems. Colloid Polym. Sci., 1998, 276, 72-80.

20. Palla, B. J. and Shah, D. O., Stabilization of high ionic strength slurries using the synergistic effects of a mixed surfactant system. J. Colloid Interface Sci., 2000, 223, 102-111. 